\title{
Interférences
}

Ars scribendi

$7 \mid 2014$

Le savoir sur la langue

\section{Y a-t-il un rapport entre les étymologies du Cratyle et les figures de son et anagrammes d'Héraclite?}

\section{Serge Mouraviev}

\section{(2) OpenEdition \\ Journals}

Édition électronique

URL : http://journals.openedition.org/interferences/4639

DOI : 10.4000/interferences.4639

ISSN : $1777-5485$

Éditeur

HiSoMA - Histoire et sources des Mondes antiques

Référence électronique

Serge Mouraviev, «Y a-t-il un rapport entre les étymologies du Cratyle et les figures de son et anagrammes d'Héraclite? », Interférences [En ligne], 7 | 2014, mis en ligne le 11 décembre 2014 consulté le 15 septembre 2020. URL : http://journals.openedition.org/interferences/4639 ; DOI : https://doi.org/10.4000/interferences.4639

Ce document a été généré automatiquement le 15 septembre 2020.

Tous droits réservés 


\title{
Y a-t-il un rapport entre les étymologies du Cratyle et les figures de son et anagrammes d'Héraclite?
}

\author{
Serge Mouraviev
}

1 Le sujet que je voulais traiter était la question de savoir si, oui ou non, et si oui dans quelle mesure, les étymologies platoniciennes du Cratyle s'apparentaient d'une façon ou d'une autre aux (combien nombreuses et diverses) figures de son et aux quelques anagrammes qu'on peut identifier chez Héraclite. Ma démarche ici n'est donc pas philosophique, encore moins est-ce une étude de la pensée platonicienne, elle est philologique et linguistique. Quand j'ai fait cette demande, je restais encore sous l'emprise d'une impression ancienne, suggérée par exemple par la comparaison

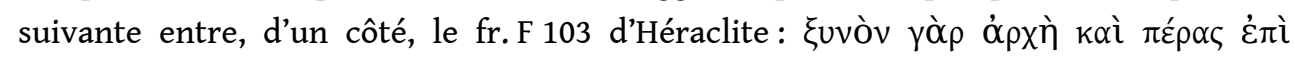

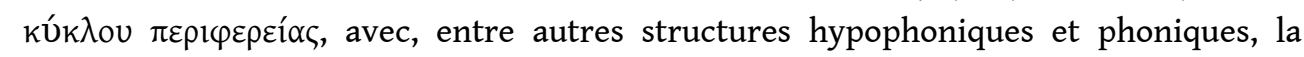

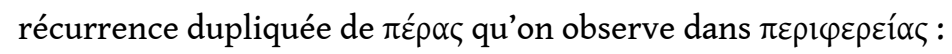

(1)

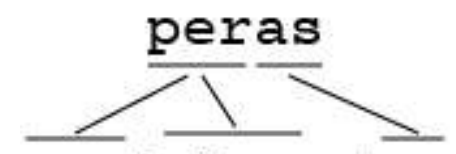

peripherejas

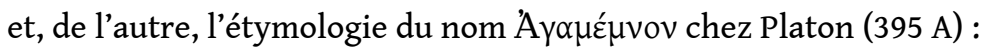


(2)

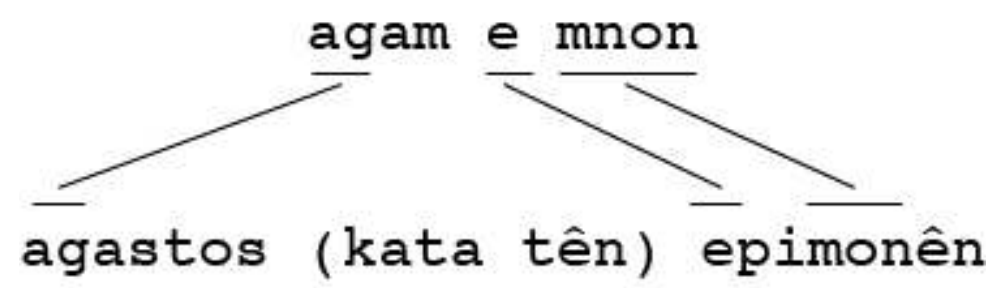

2 Il m'avait semblé que certaines des étymologies du Cratyle étaient construites selon le modèle de consonances phoniques de cette espèce - ici un anneau - ou, plus exactement (puisque les étymologies ont toujours un sens), de véritables anagrammes manifestes. Les quelques jours que j'ai pu consacrer à un réexamen plus poussé de ce sujet m'ont cependant incité à modérer cette impression première dans un sens négatif, et cela compte tenu surtout d'une différence de structure fondamentale entre les deux phénomènes. Mon résultat est donc plutôt négatif. Il n'en est pas moins, je crois, assez instructif pour l'exégèse du Cratyle.

3 Mais avant de montrer en quoi cette impression consiste, je me permettrai, pour ceux d'entre vous qui n'auraient pas eu l'occasion de consulter mes anciens travaux en la matière, de résumer brièvement les conclusions sur la poétique des fragments d'Héraclite auxquelles j'étais parvenu il y aura bientôt trente ans. Voici comment je les avais récapitulées à la fin de ma monographie de 2002 sur le langage de l'Éphésien :

Nous espérons avoir suffisamment bien montré [...] que les fragments d'Héraclite sont structurés par des configurations supralinguistiques (poétiques) à pratiquement tous les niveaux linguistiques.

$\mathrm{Au}$ niveau chronique (hypophonique) nous avons observé une rythmisation syllabotonique systématique de la totalité du texte préservé, sans parler de nombreuses incrustations quantitatives (dactylo-spondaïques et ïambotrochaïques) affectant presque la moitié de ce texte.

Au niveau phonique nous avons identifié une pléthore de consonances les plus diverses, près de quatre-vingts cas d'allitérations (simples, doubles, triples, complexes...), près de soixante-dix fragments à rimes et systèmes de rimes, un grand nombre de consonances mixtes (allitératives, rimées, en anneau, parallèles, chiasmiques, palindromiques) et de systèmes phoniques entiers, sans parler d'une dizaine d'anagrammes. - Et au niveau de l'écriture nous avons décelé des traces évidentes de configurations graphiques.

Aux niveaux morphologiques nous avons observé un recours systématique à la répétition des grammatophones, rhizophones et lexophones, créant en grand nombre des identités, accords, homonymies grammaticaux; des dérivations simples et étymologisantes et des parétymologies; des itérations, polyptotes, antanaclases, syllepses, homonomases et paronomases ( «jeux de mots» in praesentia). Nous y avons constaté aussi une utilisation régulière de ces récurrences, de concert avec les répétitions morphosémiques, pour, d'une part, construire des schèmes tactiques sophistiqués : une vingtaine de chiasmes, une trentaine de parallélismes et systèmes de parallélismes, une dizaine de schèmes mixtes et complexes combinant chiasmes, parallélismes et ordonnance palindromique; et d'autre part, produire des figures sémantiques ou tropes.

Au niveau syntaxique, nous avons noté, outre le jeu subtil des concordances ou discordances entre constructions syntaxiques et schèmes tactiques, une vingtaine d'exemples d'indétermination, de sources de polyphonies (ambiguïtés délibérées) parfois très riches allant du simple apokoïnou à la déstructuration totale.

Enfin, aux niveaux sémantiques nous avons répertorié, outre les indéterminations syntaxiques, une quarantaine d'autres polyphonies, près de quatre-vingts antithèses, 
plus de trente-cinq oxymores et paradoxes et plus de quatre-vingts comparaisons, images, exemples, énigmes et « métaphores».

Tout cela dans un corpus de 130 fragments subdivisés en près de 490 côla. particulièrement de certaines des structures linguistiques, voire supralinguistiques, observables dans les étymologies du Cratyle - desquelles structures, autant que je sache, il n'existe toujours pas d'analyse systématique, abstraction faite des problèmes d'authenticité, de sériosité et de pertinence :

- 1. les consonances mixtes parallèles, les seules qui (à la différence des consonances palindromiques ou mélangées) ne modifient pas l'ordre des éléments basiques (lettres/ sons), à ceci près, primo, que chez Héraclite ces figures ne sont pas porteuses de contenus sémantiques propres, et secundo, que celles de Platon autorisent des suppressions, ajouts ou permutations épisodiques d'éléments basiques lorsque cela ne se répercute pas trop sur la ressemblance phonique entre le nom à étymologiser et la définition proposée de son étymon;

-2. les anagrammes qui, elles, ont un contenu propre, mais dont la fonction chez Héraclite est non pas étymologique mais plutôt didactique, ou heuristique, ou griphique, ou mnémonique, ou emphatisante ;

et enfin 3. les « jeux de mots » paronomastiques quasi étymologisants.

Ces derniers sont assez rares chez Héraclite et très nombreux dans le Cratyle. Les deux exemples héraclitéens les plus éloquents sont $\mathrm{F} 48$ : le nom de l'arc est VIE mais son œuvre est MORT, où l'on pourrait soupçonner un lien étymologique entre les deux sens (accentuations) du mot BIOS (il s'agit pour Héraclite d'appuyer son identification de la

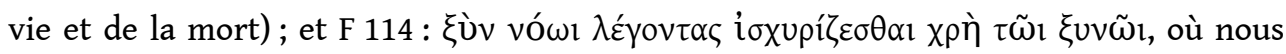
avons clairement une fausse étymologie, autrement dit une coïncidence amusante détournée à des fins rhétoriques. Pour le Cratyle, citons les multiples étymons proposés

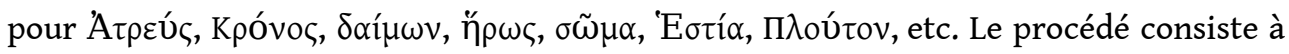
ajouter, retrancher, déplacer une ou deux lettres, pour obtenir un sens présenté comme reflétant la «vraie » signification du definiendum. La grande nouveauté est qu'il s'agit de déformer celui-ci pour le transformer en son propre definiens. Comme les déformations autorisées sont légion, la tâche est d'autant plus facile qu'est décevant le résultat. Une influence d'Héraclite, par le truchement de Cratyle, n'est pas impossible, mais comme c'est la solution la plus facile qu'on puisse imaginer, il n'est pas étonnant qu'on s'en serve chaque fois et autant de fois que cela marche.

6 S'agissant des consonances mixtes (j'appelle mixtes les consonances qui ne sont ni des allitérations, ni des rimes, ni des anneaux phoniques ou beaucoup plus que cela), elles sont de deux sortes chez Héraclite: il y a, d'un côté, les récurrences phoniques parallèles et, de l'autre, les récurrences phoniques palindromiques.

7 Prenons comme exemple le système de consonances parallèles du fr. F 90 :

(3)

\section{F 90 pyros te antamejbetaj panta | kaj pyr hapantôn | hokôsper khrysu khrêmata | kaj khrematôn khrysos.}

8 Nous y observons la récurrence parallèle simple des symphones pyr-, khrys-, khremat-, -ôn, -os, double du symphone -ant- (et simple de anta-), triple des symphones khr-, 
quadruple des phones allitératifs $p$ - et $k$-, etc., alternant dans un ordre qui n'est pas évident. Toutes ces récurrences parallèles peuvent être représentées comme ceci :

(4)

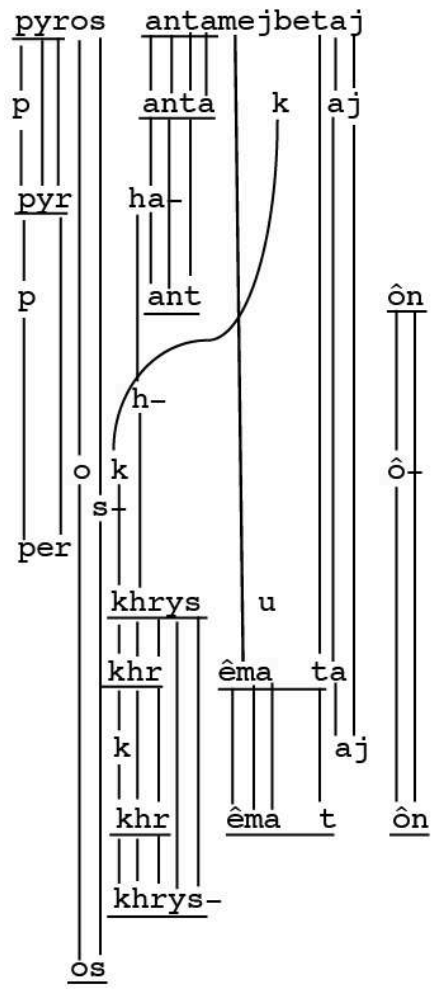

9 Un tel schéma dégage très bien les parallélismes symphoniques à l'échelle des mots, mais il ne montre que tant bien que mal la disposition tactique parallèle des symphones consonants qui précèdent hokôsper et pratiquement pas la disposition tactique chiasmique des symphones qui suivent hokôsper:

(5)

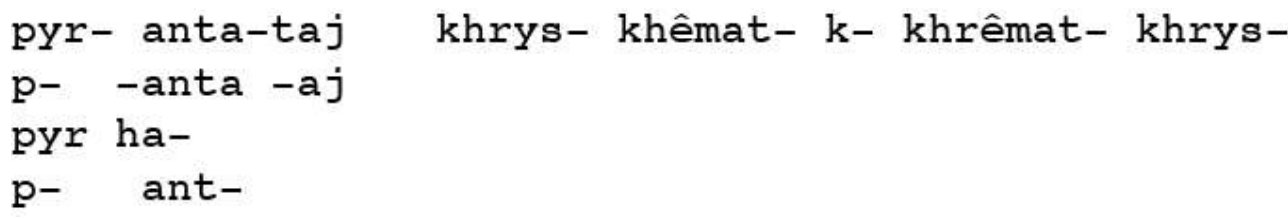

10 Et voici des exemples de consonances mixtes palindromiques, ce qui suppose que les éléments phoniques récurrents sont disposés non pas dans le même ordre que leurs « originaux » initiaux, mais dans un ordre inverse.

11 Autrement dit, si l'élément original renferme une suite de phones isolés ou couplés, l'élément corrélatif renfermera la même suite d'éléments disposés à l'envers. Ainsi, dans la rime inversée de $\mathrm{F} 1$ : 
(6)

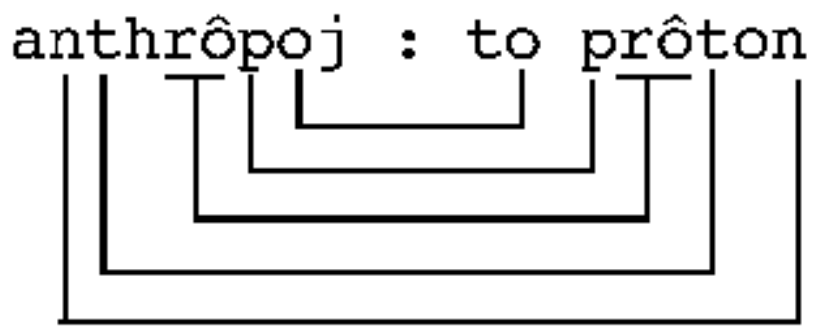

Ou dans F 14 :

(7)

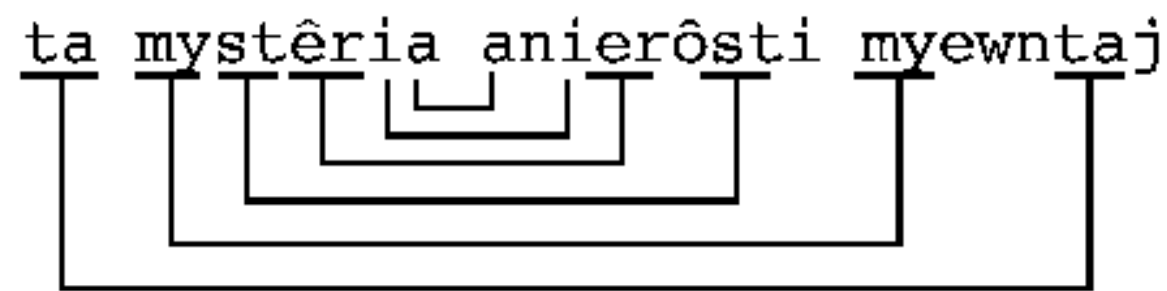

Ou encore dans F 9 :

(8)

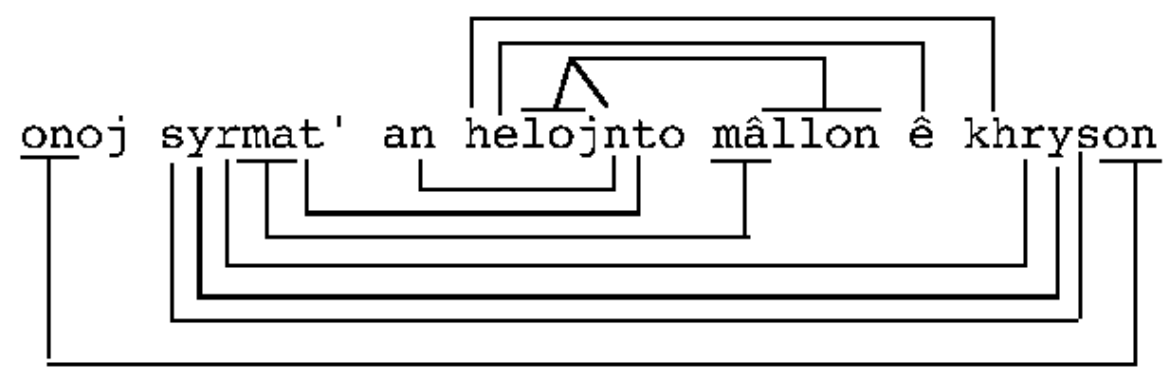

12 Le dernier exemple illustre le cas assez fréquent de double consonance palindromique : outre la configuration qui s'étend à toute la phrase, nous en observons une autre qui ne touche que sa seconde moitié. Mais si nous voulions, nous pourrions lui en préférer une autre, s'étendant, elle, à la première moitié :

(9)

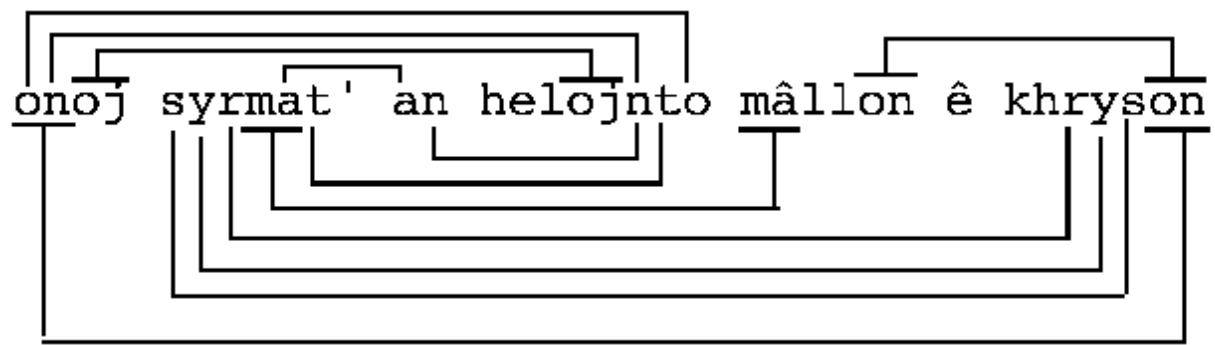

13 Si je montre ces configurations, c'est avant tout pour la beauté de la chose. Mais j'aimerais faire remarquer qu'elles aussi pourraient se prêter à des interprétations 
étymologisantes. Dans F 9 (ex. 6 ou 7), syrmat' et son corrélatif inversé (ch)ryson ou, dans F 14, mysteria et son corrélatif (an)ier(ô)st(i) se reflètent l'un l'autre en chiens de faïence. Remplacez ces couples par un etymologendum et un etymologens et vous obtiendrez une étymologie cratylienne parfaite.

Toutefois, il est douteux que Platon, l'eût-il voulu, ait pu faire appel à des structures aussi recherchées dans un dialogue en prose usuelle. Et s'il y en a dans le Cratyle, encore faudrait-il les identifier. En revanche, les structures apparentées aux consonances parallèles sont nombreuses, comme nous le verrons après un grand détour par les anagrammes.

15 F 22 nous indique exactement la procédure à suivre pour trouver l'« or » héraclitéen, pour entendre son logos : creuser beaucoup de « terre» :

(10)

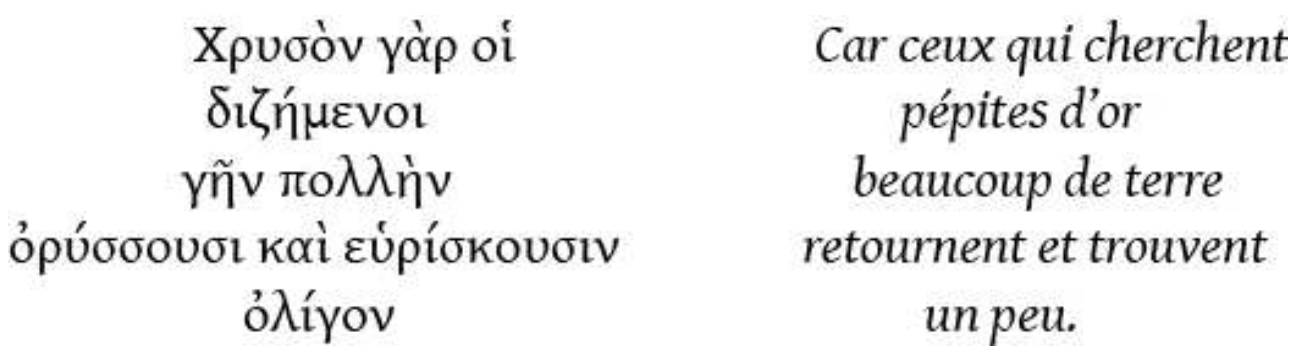

La lecture de ce fragment à haute voix permet d'en mettre en valeur la texture rythmique et phonique (sans grande prétention) : l'isosyllabie (isocolon), l'isorythmie

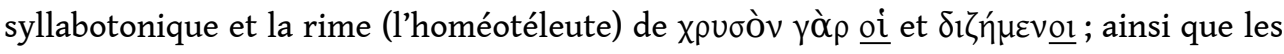

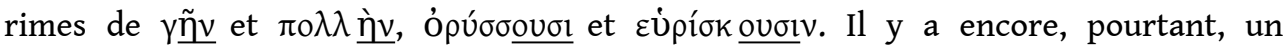
homéotéleute qui passe généralement inaperçu : $\chi \rho \cup \sigma \underline{\mathrm{o} v}$ - ỏ $\lambda \hat{i} \underline{\text { ov }}$. Or, si nous jetons un regard d'ensemble sur la totalité du fragment, nous découvrons que toutes ces rimes constituent un système symétrique parfait :

(11)

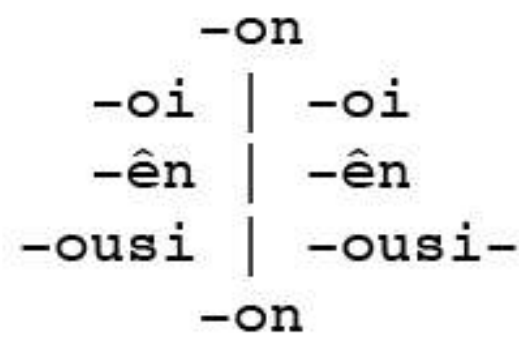

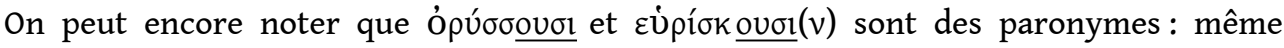
longueur, même place de l'accent, même consonantisme (à une aspiration et un

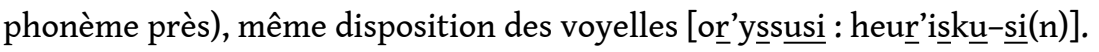

Et si nous passons maintenant du niveau phonique au niveau grammatical, nous observons ceci : 
(12)

\begin{tabular}{|c|c|c|c|}
\hline 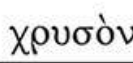 & $\gamma \grave{\alpha} \rho$ & oi & $\delta \mathrm{\iota} \zeta \eta^{\prime} \mu \varepsilon \nu o \iota$ \\
\hline Nom & Par-le. & Art & Par-pe \\
\hline $\begin{array}{l}\mathrm{m} \\
\mathrm{sg}\end{array}$ & explic. & $\underset{\mathrm{pl}}{\mathrm{m}}$ & $\underset{\mathrm{m}}{\mathrm{pl}}$ \\
\hline Acc & & Nom & Nom \\
\hline
\end{tabular}

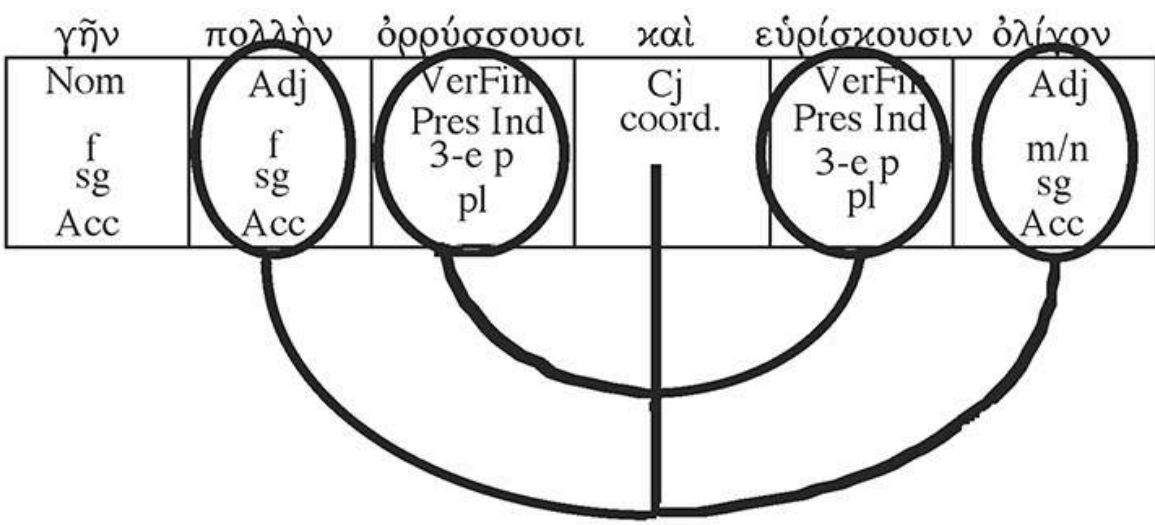

C'est-à-dire un chiasme total (sauf pour le genre) des morphosèmes grammaticaux des cinq derniers mots (la conjonction servant de pivot). Ce chiasme, nous le retrouvons au niveau des significations lexicales :

(13)

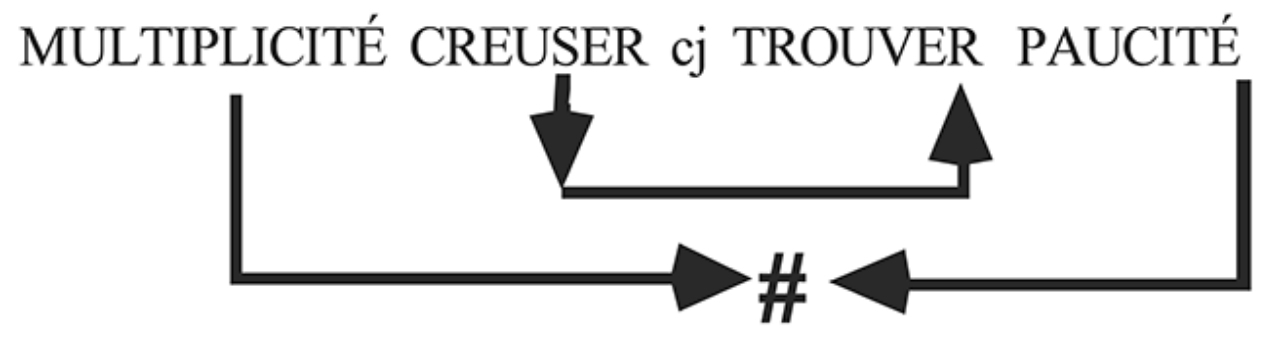

Avec toutefois cette différence que le rapport entre le premier élément (MULTIPLICITÉ) et le dernier (PAUCITÉ) se fonde non sur l'identité, mais sur la contrariété, et le rapport entre les deux éléments du milieu (CREUSEMENT et TROUVEMENT), sur la contiguïté : creuser et trouver sont deux étapes consécutives de déterrer.

Enfin, au niveau syntaxique, nous croyons retrouver toujours la même structure chiasmique du prédicat :

(14)

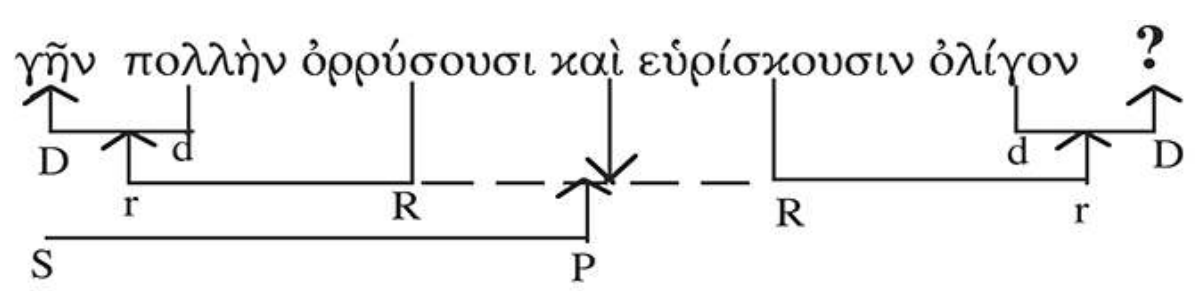


mais il nous manque un élément essentiel: le substantif déterminé par ó $\lambda$ íyov. Il est naturellement facile de combler cette ellipse. Entre $\gamma \tilde{\eta} v$ et $\chi \rho v \sigma o ́ v$, nous sommes obligés de choisir le mot qui s'accorde en genre avec ó $\lambda$ íyov. Il n'en reste pas moins que cette structure chiasmique «boiteuse » crée un sentiment de frustration syntaxique.

Seul s'en affranchira celui qui saura découvrir le mot manquant dans la texture même du fragment :

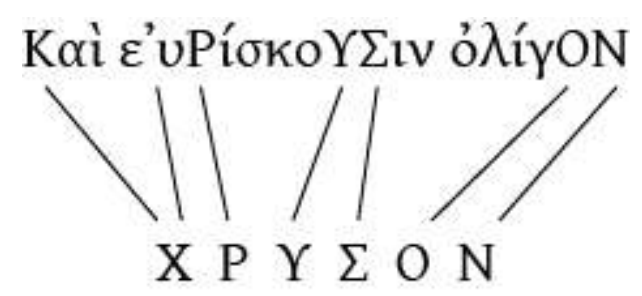

19 Ce fragment - malgré ses apparences de devinette anodine- est un modèle paradigmatique au moyen duquel Héraclite non seulement nous dit en clair que pour trouver une parcelle de vérité, de la vérité (l'image de l'or est trop transparente pour qu'on puisse douter de son sens métaphorique), il faut accomplir un grand travail, mais nous montre aussi l'objet et la méthode de ce travail : ce qu'il faut creuser, c'est le texte - celui d'Héraclite, voire celui de la réalité - et ce qu'il faut trouver et décrypter, ce sont les structures occultes qu'il dissimule.

Mais ce fragment est plus riche que cela. Il contient une seconde anagramme symétrique de la première :

\section{XPY $\triangle O N$ ГAP OI $\triangle I Z H M E N O I$ ГHN ПO $\Lambda \Lambda H N$ OPY $\bar{\Sigma} \mathrm{OY} \overline{\mathrm{I}}$

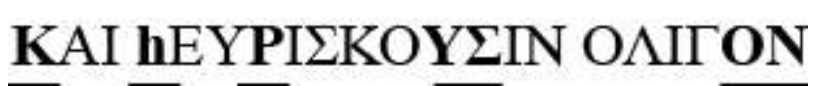

21 L'anagramme $\Gamma \mathrm{H} \mathrm{N}$ vient avant le mot $\gamma \tilde{\eta} v$ écrit en clair et supplée à l'absence d'indication explicite concernant le lieu de la recherche (le $\gamma \tilde{\eta} v$ écrit en clair désigne lui l'objet du creusement), le mot xpuбóv écrit en clair vient en tête de phrase, l'anagramme K h PY $\Sigma$ ON en queue de phrase. Ce qui crée un curieux chiasme:

(17)

\section{XPYLON Г H N ГHN K h PY $\Sigma$ O N}

un chiasme qui, du point de vue de la forme, est un parallélisme: mot en clairanagramme - mot en clair - anagramme.

Ces deux anagrammes sont des diaphones.

Malgré sa transparence, F 22 ne pose pas la question: Qui sont ces chercheurs d'or qui fouillent beaucoup de terre et trouvent peu? (réponse évidente : les amateurs de sagesse), ou: Quels sont cette terre qu'ils fouillent et cet or qu'ils cherchent? (réponse évidente : la somme des choses connaissables et la sagesse-cognition). En revanche, ce texte renferme en lui-même la réponse à deux autres questions laissées ouvertes, elles, par la syntaxe: Où cherchent les chercheurs d'or? De quoi trouvent-ils peu? Et cette réponse 
consiste non pas à dire qu'ils cherchent dans la terre (évidemment, puisque c'est elle qu'ils fouillent) et qu'ils trouvent peu d'or (évidemment, puisque ce sont des chercheurs d'or et pas d'autre chose), mais - chose beaucoup plus difficile - à trouver les mots $\Gamma H N$ et $X P Y \Sigma O N$ dans la texture même $d u$ fragment. Pour quoi faire, si les ellipses syntaxiques ne posaient aucun problème? Pour saisir la différence entre une " énigme » qui n'en est pas une et un modèle métaphorique en fonctionnement qui montre dans la matière même du verbe la façon d'extraire les mots manquants du texte, l'or de la terre, la sagesse de la réalité des choses. Résultat : nous nous retrouvons en présence d'une "façon de voir " ( $\theta \varepsilon \omega p i ́ \alpha)$ mettant en parallèle l'activité du philosophe, celle de l'orpailleur et celle du lecteur-déchiffreur attentif.

Il y a aussi chez Héraclite des anagrammes composées de symphones, et donc plus faciles à déceler acoustiquement :

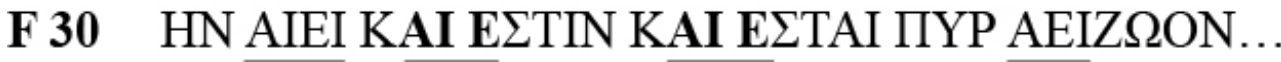

Et ici, au contraire, nous avons un triple parallélisme au niveau des morphophones et un chiasme au niveau des formes :

(19)

\section{F 15 EI KAI $\Delta$ IONY $\Sigma \Omega I \ldots$ AI $\Delta$ OIOI $\Sigma$ IN ANAI $\Delta$ E $\Sigma$ TATA $\ldots \Omega$ YTO $\Sigma \Delta$ E AI $\Delta \mathrm{H} \Sigma$ KAI $\Delta$ IONY $\Sigma \Omega \Sigma$ OTE $\Omega I \ldots \Lambda$ HNAI $\Delta \Sigma O Y \Sigma I N$}

Si dans F 30 la distinction entre anagramme et simple consonance est claire (l'anagramme est à cheval sur deux mots), dans $\mathrm{F} 15$ elle ne l'est que pour la première et la cinquième occurrences. La seconde et la troisième sont clairement des « jeux de mots » fondés sur l'homonymie des rhizosèmes (morphèmes lexicaux) 'HONTEUX', 'V ÉNÉRABLE' et 'HADÈs', la quatrième est l'occurrence de base écrite en clair et la sixième est une anagramme (c'est-à-dire une récurrence du sens caché 'HADÈs') plutôt qu'une simple consonance sans contenu sémantique en raison de l'absence de jeu de mots identifiable, de tous les cas antérieurs et du fait non négligeable que AI $\Delta$ est à cheval sur deux morphèmes $(\lambda \eta v \alpha-$ et $-1 \zeta-)$.

Signalons enfin un cas possible d'anagramme diaphonique renversée :

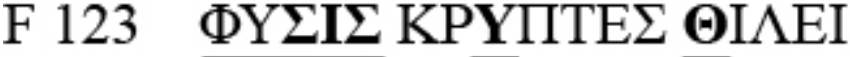

avec interpénétration du mot en clair et de l'anagramme, ce qui crée aussi une structure chiasmique : $\underline{X . Y . \Sigma I \Sigma} \underline{Y} \underline{\Phi}$.

Voilà pourquoi il faut prendre absolument au sérieux la brillante observation de Clémence Ramnoux à propos du fr. F 52. Il est vrai qu'en 1959, elle écrivait à ce propos qu' « une telle solution ne saurait être proposée elle-même que par manière de jeu ${ }^{1}$ ".

Mais l'année suivante, elle la reprend, cette fois-ci sans réserve ${ }^{2}$. En voici une version que nous avons légèrement développée : 
(21)

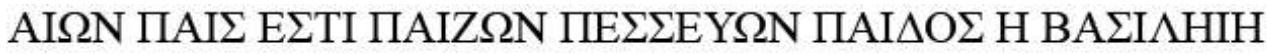

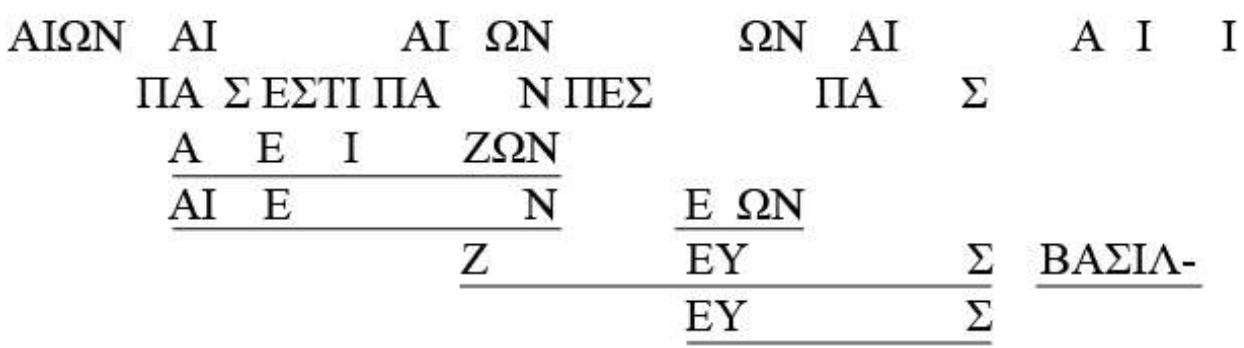

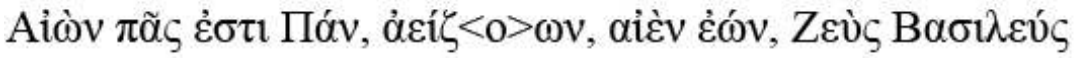
Aiôn tout entier est Pan, toujours vivant, toujours étant, Zeus-Roi

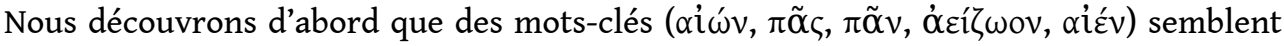
revenir dans la texture du fragment. Et nous découvrons ensuite que, pris ensemble, ils forment une espèce de formule hiératique préexistante, une formule qui semble même présenter une structure métrique :

(22)

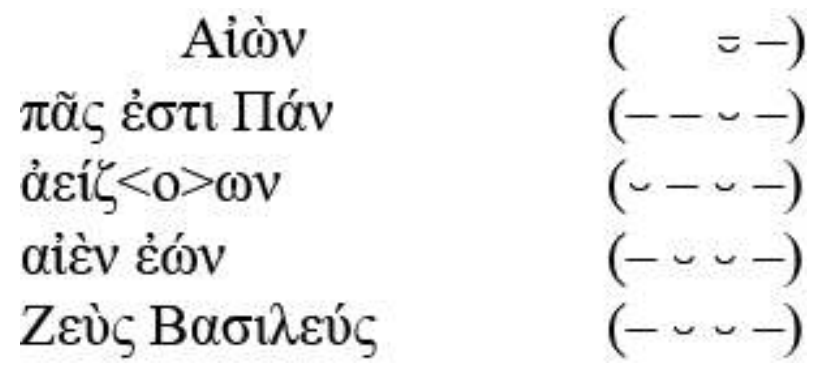

ce qui n'exclut pas la possibilité de déchiffrements légèrement différents. $\mathrm{Cl}$. Ramnoux

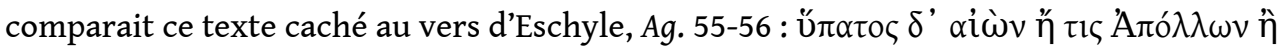
$\Pi \grave{\alpha} v$ ì Zzúc, «quelque divinité très haute, Apollon, ou Pan, ou Zeus ». Et concluait à l'existence « d'une tradition commune au moins à Héraclite et à Eschyle, pour laquelle les noms divins étaient tous à peu près équivalents pour dire le Toujours Étant ou le Toujours Vivant ${ }^{3}$ ».

Ce n'est donc pas pour rien que Clément d'Alexandrie (Paed. 1, 21, 4), parlant de la $\theta \varepsilon i ́ \alpha$

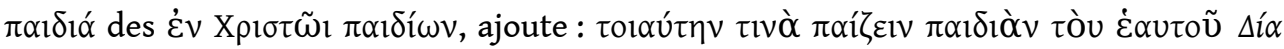

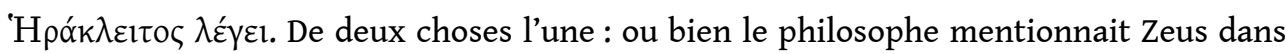
le contexte immédiat de notre citation, ou bien Clément connaissait la clé de cette énigme.

31 Et on ne manquera pas non plus de comparer l'étymologie de חó́v dans le Cratyle de

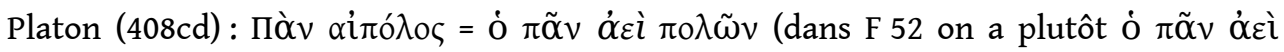

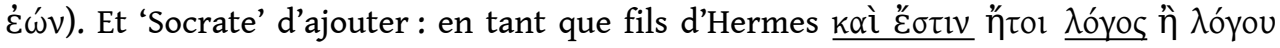

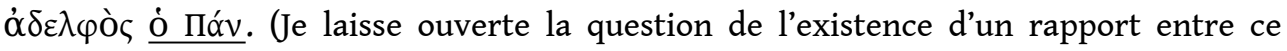
passage et notre anagramme.) 
À côté de cela, les étymologies en forme de consonances parallèles signifiantes étendues du Cratyle font piètre figure :
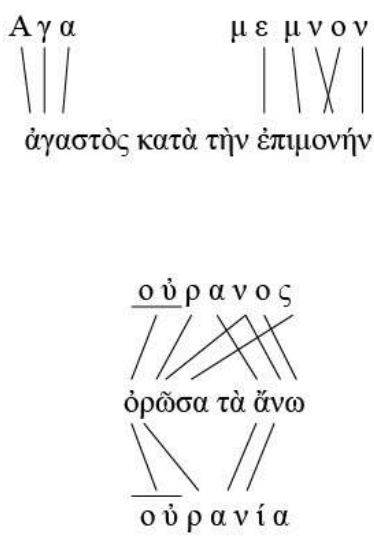

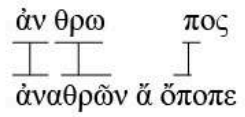

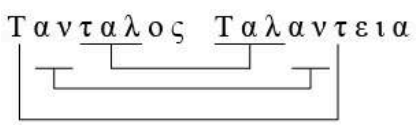

Un passage en revue assez rapide de toutes les étymologies du Cratyle (il y en a pour près de 120 mots différents dont parfois plusieurs pour le même mot) me convainc que tous les cas de ressemblance structurelle de ce genre sont plutôt des coïncidences dues au fait inéluctable que Héraclite et Platon opéraient avec des éléments identiques (les mots et les lettres-sons du grec) et, plus précisément, avec des combinaisons identiques ou ressemblantes des mêmes éléments. En revanche, leurs buts et leurs façons d'opérer étaient radicalement différents.

Platon a incontestablement commencé par réunir tout un "univers» de noms à étymologiser. Un univers structuré : d'abord les termes désignant les divinités, puis les hommes et leurs composantes (âme et corps); puis les noms des dieux du Panthéon grec dans un ordre quasi généalogique; ensuite les notions abstraites relatives à la pensée, la connaissance, la justice, la virilité / féminité, le vice et la vertu, la laideur et la beauté, la convenance et la nuisance, le plaisir et la souffrance, le désir, l'opinion et la volonté, la nécessité ; et enfin le nom, la vérité / fausseté et l'être. Pour chacun de ces noms, il cherchait (ou empruntait à la tradition) un ou plusieurs homonyme/ homoionyme appropriés (ce sont ce que j'ai appelé les «jeux de mots paronomastiques»). À défaut, il créait une courte phrase intégrant en son sein les éléments du nom étymologisé sous forme d'anagramme généralement parallèle, quelquefois avec des omissions et des permutations locales, mais jamais totalement palindromiques (l'étymologie du nom de Tantale est l'exception qui confirme la règle). Et en désespoir de cause, faisant bonne mine à mauvais jeu, il faisait appel aux deux méthodes à la fois, ou à quelque autre subterfuge encore moins convaincant, voire à 
quelque chose de tout bonnement incompréhensible. Ce faisant, il poursuivait deux buts opposés :

- 1. proposer des étymologies pour l'intégralité des noms des constituants de l'univers philosophique de son temps ;

-2. montrer au lecteur intelligent que tout cela n'était qu'un jeu et que moins une étymologie paraissait convaincante, mieux cela valait.

Le premier but exigeait :

- A. que le mot étymologisé soit phonétiquement reconnaissable dans son étymon ;

- B. que l'étymon, qu'il comporte un mot ou plusieurs, ait un sens propre conciliable avec celui du mot étymologisé.

Le deuxième but l'autorisait à faire cela "par-dessus le soulier ", sans trop se soucier de la vraisemblance et encore moins de la véracité. Seules comptaient les apparences. Pardessus le marché, il écrivait en prose, imitant le langage ordinaire de son temps.

Héraclite, au contraire, utilisait un langage extrêmement recherché, probablement hiératique, hérité de mystères auxquels il devait avoir été initié, un langage qui, pour nous, présente tous les dehors d'une prose rythmée, phonétiquement organisée, riche en tropes et en figures et rappelant par bien des côtés certaines œuvres poétiques du $\mathrm{xx}^{\mathrm{e}}$ siècle (Apollonaire, Rilke, Tsvétaïeva). Aussi n'avait-il pas besoin de sémantiser toute la partie phonétique de son discours (rythme, incrustations métriques, rimes, allitérations et consonances), ni la taxique (ordre normal vs inversion; chiasme vs parallélisme, etc.), tout cela faisant partie du style adopté. En revanche, il avait énormément besoin d'introduire des concepts nouveaux, de refléter une réalité contradictoire qu'il était seul à voir, mais ne disposait pour ce faire d'aucune terminologie appropriée, d'aucun langage spécialisé, d'aucune logique du tiers exclu... Et là, il a tiré un parti exceptionnel, en tant que moyens de modélisation, de toutes les figures phonosémiques et sémiques tout court que son langage et la variante écrite de ce langage lui offraient, y compris les « jeux de mots et de sens » (des jeux très sérieux), les figures étymologiques et les anagrammes. Conclusion: l'essentiel de ses consonances jouent un rôle quasi exclusivement esthétique (sur l'importance philosophique duquel je ne m'étendrai pas ici), tandis que ses anagrammes (sauf F 22 et $\mathrm{F}$ 15) s'apparentent parfois à des exercices de perspicacité destinés aux disciples les plus assidus. L'étymologie et la dérivation (une étymologie, une dérivation sans prétention scientifique) n'entrent en jeu que lorsqu'il s'agit de confronter des acceptions différentes, voire opposées, d'un même mot, d'un même radical, de deux homonymes et sim.

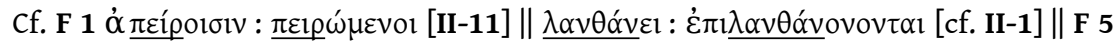

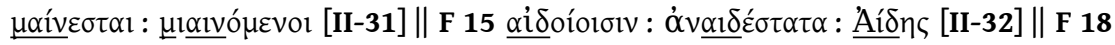

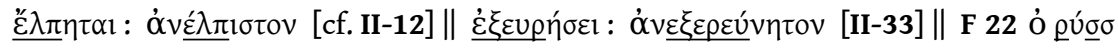

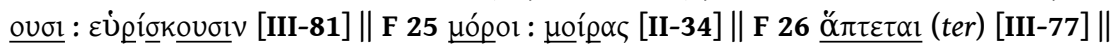

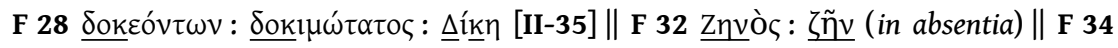

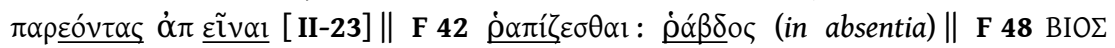

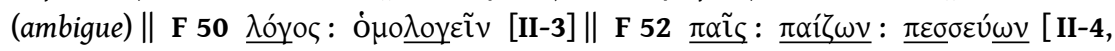

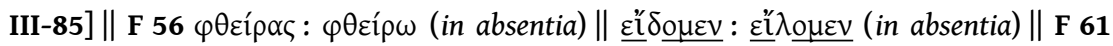

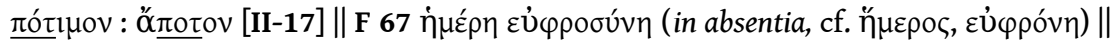

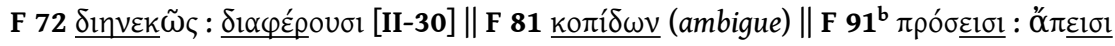

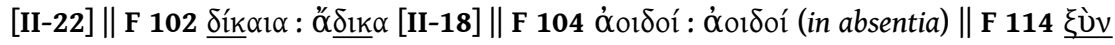




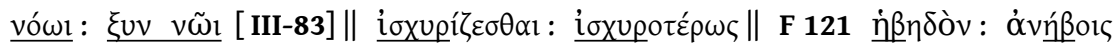

[II-14]. contenu peut - à première vue du moins - varier de négligeable (par ex. dans F 61) à fondamentale (F 114, F 48).

\section{BIBLIOGRAPHIE}

MOURAVIEV S. 2002, Heraclitea. III, Recensio : Héraclite d'Éphèse : les vestiges. 3, Les fragments du livre d'Héraclite. A, Le langage de l'Obscur. Introduction à la poétique des fragments, Sankt Augustin.

RAMNOUX Cl. 1959, Vocabulaire et structure de pensée chez Héraclite, Coll. d'études anciennes, Paris [2 ${ }^{\mathrm{e}}$ éd. : 1968].

- 1968, Héraclite ou l'homme entre les choses et les mots, $2^{\mathrm{e}}$ éd., Coll. d'études anciennes, Paris.

-1960, « Les fragments religieux », Alger = Héraclite ou l'homme entre les choses et les mots, $2^{\mathrm{e}}$ éd., Coll. d'études anciennes, Paris, 1968, p. 385-409.

\section{NOTES}

1. Ramnoux 1959, p. 449. Mais quoi de plus naturel qu'un jeu dans un texte où il est question d'un enfant qui joue?

2. Ramnoux $1960=1968$, p. 399.

3. Ramnoux 1968, p. 399.

\section{RÉSUMÉS}

Platon dépeint Cratyle comme un disciple d'Héraclite en se fondant principalement sur son adhésion à la soi-disant théorie du flux universel de ce dernier. Nulle part dans le Cratyle, ni Socrate ni Cratyle ne lui attribuent une croyance dans une correction naturelle des noms. Mais c'est un fait bien établi qu'Héraclite utilisait largement dans son livre, entre autres figures de rhétorique, des structures phoniques extrêmement élaborées, et proposait nombre de jeux de mots « étymologiques » qui suggèrent, sinon une croyance de ce type, du moins un usage délibéré des ressemblances phoniques comme moyen d'affirmer ses assertions. Beaucoup d'« étymologies » platoniciennes ressemblent aux structures purement phoniques d'Héraclite. Héraclite recourait aussi aux anagrammes pour crypter des éléments importants de son message. Pourtant, malgré ces ressemblances et analogies (qui ne sont probablement pas fortuites), les différences l'emportent largement. Tandis que Platon poursuit deux buts parallèles et opposés 
( « expliquer » les noms en les lisant à partir de leur supposé « sens original » et montrer en même temps le ridicule de ces explications), Héraclite, lui, utilise ces structures comme un élément esthétique du langage hiératique auquel il est contraint d'avoir recours, face à l'absence de quelque autre moyen plus adéquat d'expression non poétique (non lyrique ou épique) de ce qu'il considère comme nécessaire de dire, ainsi que comme un moyen d'illustrer et de modéliser sa pensée. L'étymologie et la dérivation en tant que telles et en tant que questions linguistiques ne semblent pas avoir troublé Héraclite en elles-mêmes, même si ces questions se sont présentées à lui (comme on peut le déduire de la façon dont il s'en sert), alors que chez Platon le problème est précisément de montrer que l'on ne peut absolument pas se fier à la forme de connaissance qu'elles donnent.

Cratylus is depicted by Plato as a pupil of Heraclitus mainly on account of his adherence to the latter's so called universal flux theory. Nowhere in the Cratylus do Socrates or Cratylus attribute to him a belief in a natural correctness of names. But it is an established fact that Heraclitus did make ample use in his book, among other rhetorical figures, of extremely elaborate sound patterns (rhymes, alliterations, chimes, etc.: see examples 2 through 11) and displayed a number of 'etymological' word plays suggesting if not such a belief, yet still a deliberate use of phonetic resemblances qua arguments in favour of some of his content assertions (cf. F 48, F 114, etc.). And many of Plato's 'etymologies' do indeed look like some of Heraclitus' purely phonetical sound patterns (cf. examples 1 and 2, 23). Heraclitus also resorted to anagrams as means of ciphering important elements of his message (cf. examples 12 to 22). Yet despite these (probably non fortuitous) resemblances and analogies, the differences are much more important. While Plato pursues two parallel and yet opposite goals ("explain" names by reading out of them their supposed "original meaning" and show simultaneously how ridiculous these explanations are), Heraclitus uses these patterns (a) as an aesthetic element of the hieratic language he is forced to resort to in view of the absence of any other more adequate means of non poetic (non lyric or epic) expression of what he feels necessary to say, and (b) as a means of illustrating and modelizing his meaning. Etymology and derivation as such, as linguistic problems, do not seem to have bothered Heraclitus for their own sake even if they had occurred to him (as can be inferred from the way he uses them), whereas in Plato the problem is precisely to show what unreliable sources of knowledge they are.

\section{INDEX}

Keywords : anagrams, correctness of names, derivation, epistemology, etymologies, sound patterns nomsmotscles Clément d'Alexandrie, Platon, Eschyle, Héraclite d'Éphèse

Mots-clés : anagrammes, correction des noms, dérivation, épistémologie, étymologie, structures phoniques 